\title{
Degenerate four-wave mixing in the presence of nonuniform pump wave fronts
}

\author{
Bruno Crosignani* and Amnon Yariv \\ California Institute of Technology, Pasadena, California 91125
}

Received August 6, 1983; accepted June 27, 1984

\begin{abstract}
We derive the set of coupled equations that describes the process of degenerate four-wave mixing in the presence of spatially nonuniform pump-beam wave fronts. We investigate the influence of phase mismatch between planewave pump beams on the efficiency and on the fidelity of the phase-conjugation process, and we furnish, in the near-collinear geometry, the expression of the spatial degree of coherence of the phase-conjugate wave in terms of those of the input signal and of the pump beams.
\end{abstract}

\section{INTRODUCTION}

The process of degenerate four-wave mixing in unbounded media is usually investigated by assuming the two counterpropagating pump waves to be plane waves; this approximation leads to the result that a phase-conjugate mirror can be used to obtain replicas of signals whose wave fronts have, in the paraxial approximation, an essentially arbitrary complexity. ${ }^{1}$ This restriction (obviously absent in the study of the phase-conjugation process in optical waveguides ${ }^{2-4}$ for which the two counterpropagating pump waves are identified with forward-and backward-traveling modes of the waveguide itself) has been partially removed in Ref. 5 , in which an analysis of phase-conjugate reflection has been carried out by assuming $\mathrm{TEM}_{00}$ pump beams. Recently, a detailed theory of phase-conjugation using spatially varying pump beams was published $^{6}$ that generalizes the results of Ref. 5 to a number of situations. Actually, the set of equations that is employed in Ref. 6 to describe the process of degenerate four-wave mixing with nonuniform pump and signal waves, and that constitutes the starting point of the theory, deserves, as we shall see in what follows, an accurate derivation that clarifies the conditions for its applicability and points out its limits of validity; this also, in view of different analyses of the problem, was obtained when an approach used in the frame of volume holography was adopted. ${ }^{7}$

In this paper we consider the problem of degenerate fourwave mixing in an unbounded medium using spatially nonuniform wave and derive a set of coupled integrodifferential equations that describes the process and that under suitable hypotheses reduces to the equation adopted in Ref. 6. As an application, we study the effect of a phase mismatch between the two pump beams on the process, which is shown to be influenced by the nonreciprocal self-modulation of the pump waves. Finally, we investigate the spatial-coherence properties of the phase-conjugate signal in terms of those of the pump beams and of the input signal.

The above results are obtained by employing a coupledmode formalism, which has already been used to investigate the influence of nonlinear effects ${ }^{4}$ and of imperfections ${ }^{8}$ in optical fibers.

\section{THE COUPLED-MODE FORMALISM}

By expanding the electromagnetic field in terms of the eigenmodes of an ideal guiding structure, it is possible to derive a set of first-order differential equations for the expansion coefficients, the procedure being equivalent to solving the Maxwell equations with the proper boundary conditions. ${ }^{8}$ This formalism can also be employed to investigate electromagnetic propagation in an unbounded medium, for example, the turbulent atmosphere, ${ }^{9}$ for which the appropriate set of modes is continuous.

In the context of degenerate four-wave mixing in a bulk medium (which, for the sake of simplicity, we shall assume to be isotropic), the total electric field can be written in the form

$$
\begin{aligned}
\mathbf{E}(\mathbf{r}, z, t)= & \mathbf{E}_{s}{ }^{(+)}(\mathbf{r}, z, t)+\mathbf{E}_{s}{ }^{(-)}(\mathbf{r}, z, t) \\
& +\mathbf{E}_{p}{ }^{(+)}(\mathbf{r}, z, t)+\mathbf{E}_{p}{ }^{(-)}(\mathbf{r}, z, t),
\end{aligned}
$$

where the subscripts $s$ and $p$ stand, respectively, for signal and pump (see Fig. 1). In order to apply the coupled-mode formalism to this situation, the eigenmodes of the ideal guiding structure have to be substituted for by the radiation modes with real propagation constant (which excludes the presence of evanescent waves). By assuming that the $z$ axis coincides with the (average) signal-propagation direction and that all the fields vibrate at the same angular frequency $\omega$, a possible choice is furnished by the set of orthogonally polarized states:

$$
\begin{aligned}
\mathbf{E}(\xi, 1 ; \mathbf{r})= & N_{1} \exp (-i \xi \cdot \mathbf{r})\left[\hat{x}-\left(\xi_{x} / \beta_{\xi}\right) \hat{z}\right], \\
\mathbf{E}(\xi, 2 ; \mathbf{r})= & N_{2} \exp (-i \xi \cdot \mathbf{r})\left[\left(\xi_{x} \xi_{y} / \beta_{\xi}\right) \hat{x}\right. \\
& \left.-\left(\beta_{\xi}+\xi_{x}^{2} / \beta_{\xi}\right) \hat{y}+\xi_{y} \hat{z}\right],
\end{aligned}
$$

where $\mathbf{r}=(x, y), \beta_{\xi}=\left(k^{2}-\xi^{2}\right)^{1 / 2}$ with $k=\omega n_{1} / c\left(n_{1}\right.$ being the refractive index of the medium), and the transverse wave vector $\xi$ is restricted to values of $\xi$ satisfying the relation $0 \leq$ $\xi \leq k$. The two normalization factors

$$
\begin{aligned}
& N_{1}=(1 / 2 \pi)\left[\beta_{\xi} \omega \mu_{0} /\left(\beta_{\xi}^{2}+\xi_{x}^{2}\right)\right]^{1 / 2}, \\
& N_{2}=(1 / 2 \pi)\left[\beta_{\xi} \omega \epsilon_{1} /\left(\beta_{\xi}^{2}+\xi_{x}^{2}\right)\right]^{1 / 2}
\end{aligned}
$$

have been chosen in such a way that the orthonormalization condition reads 


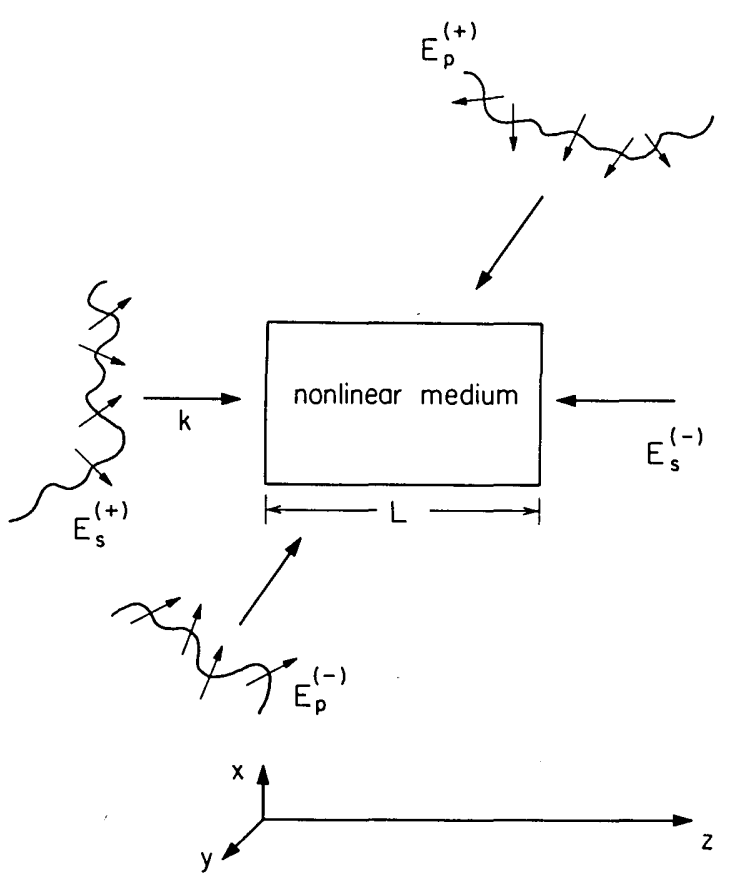

Fig. 1. Schematic geometry of degenerate four-wave mixing.

$$
\begin{array}{r}
\iint_{-\infty}^{+\infty} \mathrm{d} \mathbf{r} \hat{z} \cdot \mathbf{E}(\xi, \sigma ; \mathbf{r}) \times \mathbf{H}^{*}\left(\xi^{\prime}, \sigma^{\prime} ; \mathbf{r}\right)=\delta^{(2)}\left(\xi-\xi^{\prime}\right) \delta_{\sigma \sigma^{\prime}}, \\
\sigma, \sigma^{\prime}=1,2, \quad \mathrm{~d} \mathbf{r} \equiv \mathrm{d} x \mathrm{~d} y,
\end{array}
$$

where $\delta^{(2)}$ is the two-dimensional $\delta$ function and $\delta_{\sigma \sigma^{\prime}}$ is the Kronecker symbol.

The analytic signal of the total electric field can now be expressed in the form

$$
\begin{aligned}
\hat{\mathbf{E}}(\mathbf{r}, z, t)= & \sum_{\sigma} \iint \mathrm{d} \boldsymbol{\xi} \mathbf{E}(\xi, \sigma ; \mathbf{r})\left[c^{+}(\xi, \sigma) \exp \left(i \omega t-i \beta_{\xi} z\right)\right. \\
& \left.+c^{-}(\xi, \sigma) \exp \left(i \omega t+i \beta_{\xi} z\right)\right],
\end{aligned}
$$

where the expansion coefficients $c^{ \pm}(\xi, \sigma)$ are independent of $z$. The same expansion can be employed to study propagation in a medium possessing a generic refractive index $n(\mathbf{r}, z)$, the coefficients $c^{ \pm}(\xi, \sigma)$ becoming in this case dependent on $z$ and obeying a set of coupled differential equations, which reads

$$
\begin{aligned}
& \frac{\mathrm{d} c^{+}}{\mathrm{d} z}(\xi, \sigma ; z)=\sum_{\sigma^{\prime}} \iint \mathrm{d} \xi^{\prime}\left\{K^{+,+}\left(\xi, \sigma ; \xi^{\prime}, \sigma^{\prime} ; z\right)\right. \\
& \times \exp \left[i\left(\beta_{\xi}-\beta_{\xi^{\prime}}\right) z\right] c^{+}\left(\xi^{\prime}, \sigma^{\prime} ; z\right) \\
& \left.+K^{+,-}\left(\xi, \sigma ; \xi^{\prime}, \sigma^{\prime} ; z\right) \exp \left[i\left(\beta_{\xi}+\beta_{\xi^{\prime}}\right) z\right] c^{-}\left(\xi^{\prime}, \sigma^{\prime} ; z\right)\right\}, \\
& \frac{\mathrm{d} c^{-}}{\mathrm{d} z}(\xi, \sigma ; z)=\sum_{\sigma^{\prime}} \iint \mathrm{d} \xi^{\prime}\left\{K^{-,+}\left(\xi, \sigma ; \xi^{\prime}, \sigma^{\prime} ; z\right)\right. \\
& \times \exp \left[-i\left(\beta_{\xi}+\beta_{\xi^{\prime}}\right) z\right] c^{+}\left(\xi^{\prime}, \sigma^{\prime} ; z\right) \\
& \left.+K^{-,-}\left(\xi, \sigma ; \xi^{\prime}, \sigma^{\prime} ; z\right) \exp \left[-i\left(\beta_{\xi}-\beta_{\xi^{\prime}}\right) z\right] c^{-}\left(\xi^{\prime}, \sigma^{\prime} ; z\right)\right\} \text {, }
\end{aligned}
$$

with

$K^{p, q}\left(\xi, \sigma ; \xi^{\prime}, \sigma^{\prime} ; z\right)=p K\left(\xi, \sigma ; \xi^{\prime}, \sigma^{\prime} ; z\right)+q k\left(\xi, \sigma ; \xi^{\prime}, \sigma^{\prime} ; z\right)$, where

$$
\begin{aligned}
K\left(\xi, \sigma ; \xi^{\prime}, \sigma^{\prime} ; z\right)= & \left(\omega \epsilon_{0} / 2 i\right) \int_{-\infty}^{+\infty} \mathrm{d} \mathbf{r}\left[n^{2}(\mathbf{r}, z)-n_{1}^{2}\right] \mathbf{E}_{t} *(\xi, \sigma ; \mathbf{r}) \\
& \cdot \mathbf{E}_{t}\left(\xi^{\prime}, \sigma^{\prime} ; \mathbf{r}\right), \\
k\left(\xi, \sigma ; \xi^{\prime}, \sigma^{\prime} ; z\right)= & \left(\omega \epsilon_{0} / 2 i\right) \iint_{-\infty}^{+\infty} \mathrm{d} \mathbf{r}\left[n^{2}(\mathbf{r}, z)-n_{1}{ }^{2}\right] \mathbf{E}_{z} *(\xi, \sigma ; \mathbf{r}) \\
& \cdot \mathbf{E}_{z}\left(\xi^{\prime}, \sigma^{\prime} ; \mathbf{r}\right),
\end{aligned}
$$

and $\mathbf{E}_{t}$ and $\mathbf{E}_{z}$ indicate, respectively, the transverse and longitudinal components of the field.

Note that, up to this point, the analytical approach is completely general. The refractive-index distribution $n(\mathbf{r}$, $z$ ) is not subject to any condition of slow spatial variation, and the paraxial approximation $(\xi \ll k)$ is not required. The set of Eqs. (6) is perfectly equivalent to the Maxwell equations and does not involve the neglect of second-order derivatives with respect to $z$ of the mode amplitude, as required by the slowly varying approximation in the standard coupled-mode theory. ${ }^{10}$

\section{EVOLUTION OF THE SIGNAL AND OF THE REFLECTED BEAMS}

Let us introduce the slowly varying (with $z$ ) fields $\epsilon_{s}{ }^{\sigma, \pm}$ and $\epsilon_{p}{ }^{\sigma, \pm}$ defined by the relations

$\epsilon_{s}{ }^{\sigma, \pm}(\mathbf{r}, z)=\iint_{\Delta_{s}} \mathrm{~d} \xi \mathbf{E}(\xi, \sigma ; \mathbf{r}) \exp \left[\mp i\left(\beta_{\xi}-k\right) z\right] c^{ \pm}(\xi, \sigma ; z)$

$$
\begin{array}{r}
\epsilon_{p}^{\sigma, \pm}(\mathbf{r}, z)=\iint_{\Delta_{p}} \mathrm{~d} \xi \mathbf{E}(\xi, \sigma ; \mathbf{r}) \exp \left[ \pm i\left(\beta_{\xi}-k_{p} \pm\right) z\right] c^{ \pm} \\
\times(\xi, \sigma ; z),
\end{array}
$$

where $\Delta_{s}$ and $\Delta_{p}$ represent the intervals over which the transverse wave vectors of the signal and the pump fields, respectively, vary, and $k_{p}{ }^{ \pm}$are the (average) longitudinal wave numbers of the forward-and backward-traveling pump waves, from which it follows that

$$
\begin{aligned}
& \hat{\mathbf{E}}_{s} \pm(\mathbf{r}, z, t)=\sum_{\sigma} \epsilon_{s}{ }^{\sigma, \pm}(\mathbf{r}, z) \exp (i \omega t \mp i k z), \\
& \hat{\mathbf{E}}_{p}{ }^{ \pm}(\mathbf{r}, z, t)=\sum_{\sigma} \epsilon_{p}{ }^{\sigma, \pm}(\mathbf{r}, z) \exp \left(i \omega t \mp i k_{p}{ }^{ \pm} z\right) .
\end{aligned}
$$

By multiplying both sides of Eqs. (6a) and (6b) by $\mathbf{E}(\xi, \sigma$; $\mathbf{r}) \exp \left[-i\left(\beta_{\xi}-k\right) z\right]$ and $\mathbf{E}(\xi, \sigma ; \mathbf{r}) \exp \left[i\left(\beta_{\xi}-k\right) z\right]$, respectively, and integrating over the $\xi$ interval $\Delta_{s}$, we obtain

$$
L_{s}{ }^{+} \epsilon_{s}{ }^{\sigma,+}(\mathbf{r}, z)=\sum_{\sigma^{\prime}} \iint_{\Delta_{s}} \mathrm{~d} \xi \mathbf{E}(\xi, \sigma ; \mathbf{r})
$$

$$
\times \iint \mathrm{d} \xi^{\prime}\left(K^{+,+}\left(\xi, \sigma ; \xi^{\prime}, \sigma^{\prime} ; z\right) \exp \left[-i\left(\beta_{\xi^{\prime}}-k\right) z\right] c^{+}\left(\xi^{\prime}, \sigma^{\prime} ; z\right)\right.
$$$$
\left.+K^{+,-}\left(\xi, \sigma ; \xi^{\prime}, \sigma^{\prime} ; z\right) \exp \left[i\left(\beta_{\xi^{\prime}}+k\right) z\right] c^{-}\left(\xi^{\prime}, \sigma^{\prime} ; z\right)\right\}
$$ 
and

$$
\begin{aligned}
& L_{s}-\epsilon_{s} \sigma^{\sigma,-}(\mathbf{r}, z)=\sum_{\sigma^{\prime}} \iint_{\Delta_{s}} \mathrm{~d} \xi \mathbf{E}(\xi, \sigma ; \mathbf{r}) \\
& \quad \times \iint \mathrm{d} \xi^{\prime}\left\{K^{-,+}\left(\xi, \sigma ; \xi^{\prime}, \sigma^{\prime} ; z\right) \exp \left[-i\left(\beta_{\xi^{\prime}}+k\right) z\right] c^{+}\left(\xi^{\prime}, \sigma^{\prime} ; z\right)\right. \\
& \left.\quad+K^{-,-}-\left(\xi, \sigma ; \xi^{\prime}, \sigma^{\prime} ; z\right) \exp \left[i\left(\beta_{\xi^{\prime}}-k\right) z\right] c^{-}\left(\xi^{\prime}, \sigma^{\prime} ; z\right)\right\}
\end{aligned}
$$

where (see Appendix A)

$$
L_{s}^{ \pm}=(\partial / \partial z) \pm(i / 2 k) \nabla_{\mathbf{r}}^{2} \pm \ldots,
$$

the second- and higher-order terms on the right-hand side being negligible in the paraxial limit.

In order to maintain an analytical description that is not too complicated, we have to resort now to the paraxial approximation; this allows us to neglect in Eqs. (2) the terms containing $\xi_{x} / \beta_{\xi}$ and $\xi_{y} / \beta_{\xi}$. If we also assume that the signal field is initially polarized (along the $x$ axis), the set of equations describing the evolution of $\epsilon_{s}^{ \pm}(\mathbf{r}, z) \equiv \hat{x} \cdot \epsilon_{s}{ }^{1, \pm}(\mathbf{r}, z)$ is then easily derived from Eqs. (14) and (15), which yield

$$
\begin{aligned}
& (\partial / \partial z) \epsilon_{s}+(\mathbf{r}, z)=-\left(i k / 8 \pi^{2}\right) \iint_{-\infty}^{+\infty} \mathrm{d} \mathbf{r}^{\prime} \iint_{\Delta_{s}} \mathrm{~d} \xi \\
& \quad \times \exp \left[i \xi \cdot\left(\mathbf{r}^{\prime}-\mathbf{r}\right)\right]\left\{\left[n^{2}\left(\mathbf{r}^{\prime}, z\right) / n_{1}{ }^{2}\right]-1\right\} \\
& \quad \times\left\{\epsilon_{s}+\left(\mathbf{r}^{\prime}, z\right)+\exp \left[i\left(k-k_{p}+\right) z\right] \epsilon_{p}+\left(\mathbf{r}^{\prime}, z\right)\right. \\
& \left.\quad+\exp (2 i k z) \epsilon_{s}-\left(\mathbf{r}^{\prime}, z\right)+\exp \left[i\left(k+k_{p}-\right)\right] \epsilon_{p}-\left(\mathbf{r}^{\prime}, z\right)\right\}
\end{aligned}
$$

and

$$
\begin{aligned}
& (\partial / \partial z) \epsilon_{s}-(\mathbf{r}, z)=\left(i k / 8 \pi^{2}\right) \iint_{-\infty}^{+\infty} \mathrm{d} \mathbf{r}^{\prime} \int_{\Delta_{s}} \mathrm{~d} \xi \\
& \left.\quad \times \exp \left[i \xi \cdot\left(\mathbf{r}^{\prime}-\mathbf{r}\right)\right]\left\{n^{2}\left(\mathbf{r}^{\prime}, z\right) / n_{1}{ }^{2}\right]-1\right\} \\
& \quad \times\left\{\epsilon_{s}-\left(\mathbf{r}^{\prime}, z\right)+\exp \left[i\left(k-k_{p}-\right) z\right] \epsilon_{p}-\left(\mathbf{r}^{\prime}, z\right)\right. \\
& \left.\quad+\exp (-2 i k z) \epsilon_{s}{ }^{+}\left(\mathbf{r}^{\prime}, z\right)+\exp \left[-i\left(k+k_{p}{ }^{+}\right) z\right] \epsilon_{p}{ }^{+}\left(\mathbf{r}^{\prime}, z\right)\right\}
\end{aligned}
$$

where $\epsilon_{p}{ }^{+}(\mathbf{r}, z) \equiv \hat{x} \cdot \epsilon_{p}{ }^{1, \pm}(\mathbf{r}, z)$.

We shall now assume that the optical Kerr effect is responsible for the process of four-wave mixing and write, accordingly,

$$
n(\mathbf{r}, z)=n_{1}+n_{2}|\hat{E}(\mathbf{r}, z, t)|^{2},
$$

where $n_{2}$ is the nonlinear refractive-index constant of the medium and where, recalling Eqs. (1), (12), and (13),

$$
\begin{aligned}
& |\hat{E}(\mathbf{r}, z, t)|^{2}=\left|\epsilon_{s}{ }^{+}\right|^{2}+\left|\epsilon_{s}-\right|^{2}+\left|\epsilon_{p}+\right|^{2}+\left|\epsilon_{p}-\right|^{2} \\
& \quad+\left\{\exp (-2 i k z) \epsilon_{s}{ }^{+} \epsilon_{s}-*+\exp \left[-i\left(k+k_{p}-\right) z\right] \epsilon_{s}{ }^{+} \epsilon_{p}-*\right. \\
& \quad+\exp \left[i\left(k_{p}{ }^{+}-k\right) z\right] \epsilon_{s}{ }^{+} \epsilon_{p}+*+\exp \left[i\left(k+k_{p}+\right) z\right] \epsilon_{s}-\epsilon_{p}+* \\
& \quad+\exp \left[i\left(k-k_{p}-\right) z\right] \epsilon_{s}-\epsilon_{p}-* \\
& \left.\quad+\exp \left[-i\left(k_{p}+k_{p}-\right) z\right] \epsilon_{p}{ }^{+} \epsilon_{p}-*+\text { c.c. }\right\}
\end{aligned}
$$

[in writing Eq. (20) we have taken advantage of the hypothesis that $\left|E_{p z}\right|^{2} \ll\left|E_{p x}\right|^{2}$ and that $\left|E_{p y}\right|^{2} \ll\left|E_{p x}\right|^{2}$. By inserting Eq. (19) into Eqs. (17) and (18) and by keeping on the right-hand sides of the resulting equations only the terms that do not contain any fast $z$ dependence of the kind $\exp ( \pm 2 i k z)$, $\exp \left[ \pm 2 i\left(k_{p}{ }^{+}+k_{p}{ }^{-}\right) z\right]$, or $\exp \left[i\left(k \pm k_{p}{ }^{ \pm}\right) z\right]$, and by allowing for a small phase mismatach $\Delta k=k_{p}{ }^{-}-k_{p}{ }^{+}$of the pump waves, we finally get

$$
\begin{aligned}
& (\partial / \partial z) \epsilon_{s}^{+}(\mathbf{r}, z)=\left(-i a / 4 \pi^{2}\right) \iint_{-\infty}^{+\infty} \mathrm{d} \mathbf{r}^{\prime} \iint_{\Delta_{s}} \mathrm{~d} \xi \\
& \quad \times \exp \left[i \xi \cdot\left(\mathbf{r}^{\prime}-\mathbf{r}\right)\right]\left\{2 \left[\left|\epsilon_{p}{ }^{+}\left(\mathbf{r}^{\prime}, z\right)\right|^{2}\right.\right. \\
& \left.\quad+\left|\epsilon_{p}{ }^{-}\left(\mathbf{r}^{\prime}, z\right)\right|^{2}\right] \epsilon_{s}^{+}\left(\mathbf{r}^{\prime}, z\right) \\
& \quad+\left[2\left|\epsilon_{s}-\left(\mathbf{r}^{\prime}, z\right)\right|^{2}+\left|\epsilon_{s}{ }^{+}\left(\mathbf{r}^{\prime}, z\right)\right|^{2}\right] \epsilon_{s}{ }^{+}\left(\mathbf{r}^{\prime}, z\right) \\
& \left.\quad+2 \exp (i \Delta k z) \epsilon_{p}-\left(\mathbf{r}^{\prime}, z\right) \epsilon_{p}{ }^{+}\left(\mathbf{r}^{\prime}, z\right) \epsilon_{s}^{-*}\left(\mathbf{r}^{\prime}, z\right)\right\},
\end{aligned}
$$

where we have set $a=k n_{2} / n_{1}$, a perfectly analogous equation holding true for $\epsilon_{s}{ }^{-}$, provided that one performs on the right-hand side of Eq. (21) the change $+\rightarrow-,-\rightarrow+$, and $a$ $\rightarrow-a$.

By inspecting Eq. (21), we recognize that if the quantities $\left|\epsilon_{p} \pm\left(\mathbf{r}^{\prime}, z\right)\right|^{2}$ and $\epsilon_{p}-\left(\mathbf{r}^{\prime}, z\right) \epsilon_{p}{ }^{+}\left(\mathbf{r}^{\prime}, z\right)$ do not contain, as a function of $\mathbf{r}^{\prime}$, spatial frequencies outside the interval $\Delta_{s}$, the right-hand side of Eq. (21) consists of a space Fourier transform followed by the inverse operation, so that, in this case, one can write (assuming that $\left|\epsilon_{s}{ }^{ \pm}\right|^{2} \ll\left|\epsilon_{p}{ }^{ \pm}\right|^{2}$ )

$$
\begin{gathered}
(\partial / \partial z) \epsilon_{s}{ }^{+}(\mathbf{r}, z)=-2 i a\left[\left|\epsilon_{p}{ }^{+}(\mathbf{r}, z)\right|^{2}+\left|\epsilon_{p}-(\mathbf{r}, z)\right|^{2}\right] \epsilon_{s}{ }^{+}(\mathbf{r}, z) \\
-2 i a \exp (i \Delta k z) \epsilon_{p}{ }^{-}(\mathbf{r}, z) \epsilon_{p}{ }^{+}(\mathbf{r}, z) \epsilon_{s}-*(\mathbf{r}, z) .
\end{gathered}
$$

An analogous expression, obtained by performing the changes specified above is valid for $\epsilon_{s}-$.

Note that, if we assume that

$$
\begin{aligned}
& \epsilon_{p}{ }^{+}(\mathbf{r}, z)=\exp \left(-i \xi^{+} \cdot \mathbf{r}\right) f_{p}{ }^{+}(\mathbf{r}, z), \\
& \epsilon_{p}{ }^{-}(\mathbf{r}, z)=\exp \left(i \xi^{-} \cdot \mathbf{r}\right) f_{p}-(\mathbf{r}, z),
\end{aligned}
$$

where $\xi^{ \pm 2}=k^{2}-k_{p}^{ \pm 2}$ and where the $f_{p}^{ \pm}(\mathbf{r}, z)$ are slowly varying functions (the ideal case of exactly contradirectional plane-wave pump waves corresponding to $f_{p}{ }^{ \pm}$constant and $\xi^{+}=\xi^{-}$), the above conditions require, in particular, that $\Delta \xi$ $=\xi^{-}-\xi^{+}$not lie outside $\Delta_{s}$.

\section{EVOLUTION OF THE PUMP BEAMS}

The set consisting of Eq. (22) and of the analogous one for $\epsilon_{s}-$ needs, in order to be solved, to be supplemented with the equations describing the evolution of the pump beams. With a procedure completely analogous to that leading to Eq. (22) and under the same hypotheses, it is possible to show that $f_{p}+(\mathbf{r}, z)$ obeys the equation

$$
L_{p}{ }^{+} f_{p}+(\mathbf{r}, z)=-i a\left[\left|f_{p}+(\mathbf{r}, z)\right|^{2}+2\left|f_{p}-(\mathbf{r}, z)\right|^{2}\right] f_{p}+(\mathbf{r}, z),
$$

where

$$
L_{p}{ }^{+}=(\partial / \partial z)+\left[\left(\xi^{+} / k_{p}{ }^{+}\right) \cdot \nabla_{\mathbf{r}}\right] .
$$

The equation obeyed by $f_{p}-(\mathbf{r}, z)$ is obtainable with the usual changes, provided that one writes

$$
L_{p}-=(\partial / \partial z)+\left[\left(\xi^{-/ k_{p}}-\right) \cdot \nabla_{\mathbf{r}}\right]
$$

Equations (24) and (25) and the analogous equations for $\epsilon_{s}$ and $f_{p}{ }^{-}$completely describe the process of four-wave mixing. However, it is evident that the equations for $f_{p}{ }^{+}$and $f_{p}{ }^{-}$can often be most conveniently rewritten, in view of the boundary conditions imposed on them, in coordinate systems that have one of their axes (say, $z^{\prime}$ and $z^{\prime \prime}$ ) aligned with the vectors $\left(\xi^{+}\right.$, $\left.k_{p}{ }^{+}\right)$and $\left(\xi^{-}, k_{p}{ }^{-}\right)$, respectively. If one refers, for example, to Eq. (24), one can achieve the rewriting by passing from the umprimed coordinate system characterized by the three 
mutually orthogonal unit vectors $\hat{e}_{x}, \hat{e}_{y}$, and $\hat{e}_{z}$ to one described by the unit vectors $\hat{e}_{x^{\prime}}^{\prime}, \hat{e}_{y^{\prime}}$, and $\hat{e}_{z^{\prime}}$ by means of the change of variables

$$
\begin{aligned}
& x^{\prime}=x\left(\hat{e}_{x^{\prime}} \cdot \hat{e}_{x}\right)+y\left(\hat{e}_{x^{\prime}} \cdot \hat{e}_{y}\right)+z\left(\hat{e}_{x^{\prime}} \cdot \hat{e}_{z}\right), \\
& y^{\prime}=x\left(\hat{e}_{y^{\prime}} \cdot \hat{e}_{x}\right)+y\left(\hat{e}_{y^{\prime}} \cdot \hat{e}_{y}\right)+z\left(\hat{e}_{y^{\prime}} \cdot \hat{e}_{z}\right), \\
& z^{\prime}=x\left(\hat{e}_{z^{\prime}} \cdot \hat{e}_{x}\right)+y\left(\hat{e}_{z^{\prime}} \cdot \hat{e}_{y}\right)+z\left(\hat{e}_{z^{\prime}} \cdot \hat{e}_{z}\right),
\end{aligned}
$$

provided that $\hat{e}_{z^{\prime}}$ fulfills the conditions

$$
\hat{e}_{z^{\prime}} \cdot \hat{e}_{x}=\xi_{x}+/ k, \quad \hat{e}_{z^{\prime}} \cdot \hat{e}_{y}=\xi_{y}+/ k, \quad \hat{e}_{z^{\prime}} \cdot \hat{e}_{z}=k_{p}+/ k .
$$

A relation completely similar to Eqs. (28) is valid for the double-primed variables referring to the equation describing $f_{p}{ }^{-}, \hat{e}_{z^{\prime \prime}}$ being in this case determined by the conditions

$$
\hat{e}_{z^{\prime \prime}} \cdot \hat{e}_{x}=\xi_{x}-/ k, \quad \hat{e}_{z^{\prime \prime}} \cdot \hat{e}_{y}=\xi_{y}-/ k, \quad \hat{e}_{z^{\prime \prime}} \cdot \hat{e}_{z}=k_{p}-/ k .
$$

In these coordinate systems, the equations describing the evolution of $f^{+}$and $f_{p}$ - become

$$
\begin{aligned}
& \left(\partial / \partial z^{\prime}\right) f_{p}{ }^{+}\left(\mathbf{r}^{\prime}, z^{\prime}\right) \\
& \quad=-i a\left[\left|f_{p}{ }^{+}\left(\mathbf{r}^{\prime}, z^{\prime}\right)\right|^{2}+2 \mid f_{p}{ }^{\left.-\left.\left(\mathbf{r}^{\prime}, z^{\prime}\right)\right|^{2}\right] f_{p}+\left(\mathbf{r}^{\prime}, z^{\prime}\right)}\right.
\end{aligned}
$$

and

$$
\begin{aligned}
& \left(\partial / \partial z^{\prime \prime}\right) f_{p}-\left(\mathbf{r}^{\prime \prime}, z^{\prime \prime}\right) \\
& \quad=i a\left[2\left|f_{p}{ }^{+}\left(\mathbf{r}^{\prime \prime}, z^{\prime \prime}\right)\right|^{2}+\left|f_{p}-\left(\mathbf{r}^{\prime \prime}, z^{\prime \prime}\right)\right|^{2}\right] f_{p}-\left(\mathbf{r}^{\prime \prime}, z^{\prime \prime}\right) .
\end{aligned}
$$

\section{INFLUENCE OF PHASE MISMATCH BETWEEN THE PUMP WAVES}

If we assume that the two pump beams are represented by plane waves, Eqs. (30) and (31) can be integrated immediately to give

$$
\begin{aligned}
f_{p}{ }^{+}\left(\mathbf{r}^{\prime}, z^{\prime}\right) & \equiv f_{p}^{+}\left(z^{\prime}\right) \\
& =\exp \left[-i a\left(I^{+}+2 I^{-}\right) z^{\prime}\right] f_{p}+\left(z^{\prime}=0\right)
\end{aligned}
$$

and

$$
\begin{aligned}
f_{p}-\left(\mathbf{r}^{\prime \prime}, z^{\prime \prime}\right) & \equiv f_{p}-\left(z^{\prime \prime}\right) \\
& =\exp \left[i a\left(2 I^{+}+I^{-}\right)\left(z^{\prime \prime}-L\right)\right] f_{p}-\left(z^{\prime \prime}=L\right),
\end{aligned}
$$

where $L$ is the interaction length of the pump waves, $I^{+} \equiv$ $\left|f_{p}{ }^{+}\left(z^{\prime}=0\right)\right|^{2}$, and $I^{-} \equiv\left|f_{p}-\left(z^{\prime \prime}=L\right)\right|^{2}$. Equations (32) and (33) allow us, by means of Eqs. (27)-(29), to deduce the relation

$$
\begin{array}{r}
\exp (i \Delta k z) \epsilon_{p}-(\mathbf{r}, z) \epsilon_{p}+(\mathbf{r}, z)=\exp \left[-i a\left(2 I^{+}+I^{-}\right) L\right. \\
+(i \delta \xi \cdot \mathbf{r})+i \delta k z] f_{p}+\left(z^{\prime}=0\right) f_{p}-\left(z^{\prime \prime}=L\right)
\end{array}
$$

where

$$
\delta \xi=\xi^{-}\left[1+(a / k)\left(2 I^{+}+I^{-}\right)\right]-\xi^{+}\left[1+(a / k)\left(I^{+}+2 I^{-}\right)\right]
$$

and

$\delta k=k_{p}-\left[1+(a / k)\left(2 I^{+}+I^{-}\right)\right]-k_{p}+\left[1+(a / k)\left(I^{+}+2 I^{-}\right)\right]$.

By inserting Eqs. (32)-(34) into Eq. (22) and into the analogous equation for $\epsilon_{s}^{-}$, we finally obtain in the paraxial approximation $(\partial / \partial z) \epsilon_{s}{ }^{+}(\mathbf{r}, z)=-i B \epsilon_{s}{ }^{+}(\mathbf{r}, z)-i D(\mathbf{r}) \exp (i \delta k z) \epsilon_{s}{ }^{-*}(\mathbf{r}, z)$, $(\partial / \partial z) \epsilon_{s}^{-*}(\mathbf{r}, z)=-i B \epsilon_{s}^{-*}(\mathbf{r}, z)$

$$
-i D^{*}(\mathbf{r}) \exp (-i \delta k z) \epsilon_{s}{ }^{+}(\mathbf{r}, z) \text {, }
$$

where

$$
\begin{aligned}
B= & 2 a\left(I^{+}+I^{-}\right), \\
D(\mathbf{r})= & 2 a \exp \left[-i a\left(2 I^{+}+I^{-}\right) L+(i \delta \xi \cdot \mathbf{r})\right] \\
& \times f_{p}{ }^{+}\left(z^{\prime}=0\right) f_{p}{ }^{-}\left(z^{\prime \prime}=L\right) .
\end{aligned}
$$

The set of Eqs. (37) can be easily integrated, and its solution, which obeys the boundary conditions $\epsilon_{s}{ }^{+}(\mathbf{r}, z=0)=$ $\epsilon_{s}{ }^{+}(\mathbf{r}, 0)$ and $\epsilon_{s}{ }^{-}(\mathbf{r}, z=L)=0$, reads

$$
\begin{aligned}
& \epsilon_{s}{ }^{+}(\mathbf{r}, z)=\exp (-i B z+i \alpha z) \\
& \times \frac{\gamma^{2} \cos [\gamma(L-z)]+i \alpha \gamma \sin [\gamma(L-z)]}{\gamma^{2} \cos (\gamma L)+i \alpha \sin (\gamma L)} \epsilon_{s}{ }^{+}(\mathbf{r}, 0), \\
& \epsilon_{s}^{-*}(\mathbf{r}, z)=-i \exp (-i B z-i \alpha z) \\
& \times \frac{\sin [\gamma(z-L)]}{\gamma \cos (\gamma L)+i \alpha \sin (\gamma L)} D^{*}(\mathbf{r}) \epsilon_{s}{ }^{+}(\mathbf{r}, 0),
\end{aligned}
$$

with $\alpha=\delta k / 2$ and $\gamma=\left(|D|^{2}+\alpha^{2}\right)^{1 / 2}$. In particular, at $z=0$ we obtain

$$
\epsilon_{s}-(\mathbf{r}, 0)=-i D(\mathbf{r}) \frac{\sin (\gamma L)}{\gamma \cos (\gamma L)+i \alpha \sin (\gamma L)} \epsilon_{s}+*(\mathbf{r}, 0),
$$

which shows that the influence of the phase mismatch $(\delta k \neq$ 0 ) on the phase-conjugation process consists of both a reduction in its efficiency,

$$
\begin{aligned}
R= & \frac{\mid \epsilon_{s}-\left(\mathbf{r},\left.0\right|^{2}\right.}{\left|\epsilon_{s}{ }^{+}(\mathbf{r}, 0)\right|^{2}}=\frac{|D|^{2}}{|D|^{2}+\alpha^{2}} \\
& \times \frac{\tan ^{2}\left[\left(|D|^{2}+\alpha^{2}\right)^{1 / 2} L\right]}{1+\left[\alpha^{2} /\left(|D|^{2}+\alpha^{2}\right)\right] \tan ^{2}\left[\left(|D|^{2}+\alpha^{2}\right)^{1 / 2} L\right]},
\end{aligned}
$$

and the appearance of a phase factor $\exp (i \delta \xi \cdot \mathbf{r})$ that alters the phase of the reflected signal $\epsilon_{s}{ }^{-}(\mathbf{r}, 0)$ and thus deteriorates the fidelity of the process.

The power-reflection coefficient $R$ formally coincides with that worked out for ideal degenerate four-wave mixing ${ }^{11}$ (plane-wave signal and pump beams, no phase mismatch) in the presence of wavelength detuning (see Fig. 2), provided that one identifies $\kappa$ with $|D|$ and $\Psi$ with $\delta k L / 2 \pi$.

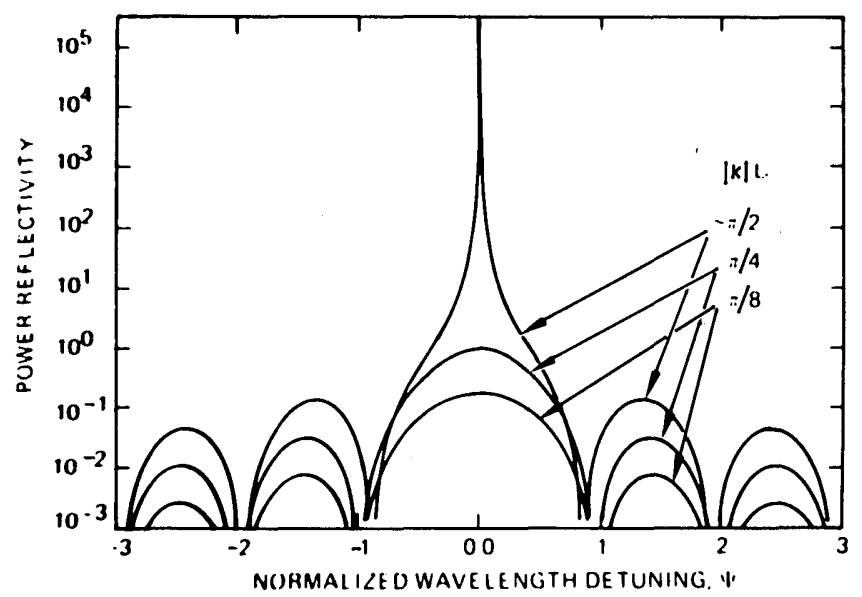

Fig. 2. Power reflectivity $R$ versus normalized wavelength detuning $\Psi$ for several values of the nonlinear gain $|\kappa| L$ (after Ref. 11). 


\section{SPATIAL COHERENCE OF THE REFLECTED SIGNAL}

In the above considerations, the electric field has been assumed to be perfectly deterministic. However, it is to be expected that, in practical situations, both the pump and the signal waves will exhibit some degree of randomness, so that the description in the above sections concerns only the single realization of the electric field, which is actually a stochastic variable. Accordingly, the meaningful quantity is not $\epsilon_{s}-(\mathbf{r}$, 0 ) itself but rather its mutual coherence function $\Gamma\left(\mathbf{r}, \mathbf{r}^{\prime}\right)$, defined as

$$
\Gamma\left(\mathbf{r}, \mathbf{r}^{\prime}\right)=\left\langle\epsilon_{s}^{-}(\mathbf{r}, 0) \epsilon_{s}^{-*}\left(\mathbf{r}^{\prime}, 0\right)\right\rangle,
$$

where the angular brackets stand for an ensemble average over its possible realizations or, in stationary situations, for time average over an interval that is long compared with the typical time scale of fluctuations.

In order to be able to evaluate explicitly the expression appearing in Eq. (43), we assume a simple, nearly collinear geometry in which the pump, the signal, and the reflected beams overlap almost completely. Furthermore, we suppose that the spatial incoherence of the pump waves is associated with a randomly varying position-dependent phase, that is, $f_{p}{ }^{+}(\mathbf{r}, z=0)=\left(I^{+}\right)^{1 / 2} \exp \left[i \phi_{p}+(\mathbf{r})\right], f_{p}-(\mathbf{r}, z=L)=\left(I^{-}\right)^{1 / 2}$ $\exp \left[i \phi_{p}-(\mathbf{r})\right]$, an assumption apt to represent the behavior of an amplitude-stabilized laser field. The signal field is written in the form $\epsilon_{s}{ }^{+}(\mathbf{r}, 0)=\exp \left[i \phi_{s}(\mathbf{r})\right] S(\mathbf{r})$, where $\phi_{s}(\mathbf{r})$ represents both the intrinsic phase fluctuations of the source and those resulting, for example, from its passage through a random medium (the distortions of which are to be corrected by the phase-conjugation process).

In the paraxial approximation, with a procedure similar to the one followed in the previous section, it is easy to obtain

$$
\begin{aligned}
\epsilon_{s}-(\mathbf{r}, 0)= & -i \exp \left[-i a\left(2 I^{+}+I^{-}\right) L\right] \exp \left[i \phi_{p}+(\mathbf{r})+i \phi_{p}-(\mathbf{r})\right] \\
& \times \tan \left[2 a\left(I^{+} I^{-}\right)^{1 / 2} L\right] \exp \left[-i \phi_{s}(\mathbf{r})\right] S^{*}(\mathbf{r})
\end{aligned}
$$

and

$$
\begin{aligned}
\Gamma\left(\mathbf{r}, \mathbf{r}^{\prime}\right)= & \tan ^{2}\left[2 a\left(I^{+} I^{-}\right)^{1 / 2} L\right] \\
& \times\left\langle\exp \left[i \phi_{p}{ }^{+}(\mathbf{r})+i \phi_{p}-(\mathbf{r})-i \phi_{s}(\mathbf{r})\right]\right. \\
& \times \exp \left[-i \phi_{p}{ }^{+}\left(\mathbf{r}^{\prime}\right)-i \phi_{p}{ }^{-}\left(\mathbf{r}^{\prime}\right)\right. \\
& \left.\left.+i \phi_{s}\left(\mathbf{r}^{\prime}\right)\right]\right\rangle S^{*}(\mathbf{r}) S\left(\mathbf{r}^{\prime}\right) .
\end{aligned}
$$

If we assume that the signal and the pump fields are generated by the same laser source and indicate with $\Psi(\mathbf{r})$ the extra phase fluctuation of the signal resulting from its passage through a random medium, then, supposing that all the mutual time delays of the various beams are short compared with the coherence time of the source, we can write $\phi_{p}+(\mathbf{r})=$ $\phi_{p}-(\mathbf{r}) \equiv \phi(\mathbf{r}), \phi_{s}(\mathbf{r})=\phi(\mathbf{r})+\Psi(\mathbf{r})$. Since $\phi(\mathbf{r})$ and $\Psi(\mathbf{r})$ are statistically independent, we finally obtain

$$
\begin{aligned}
\Gamma\left(\mathbf{r}, \mathbf{r}^{\prime}\right)= & \tan ^{2}\left[2 a \eta^{2}\left(I^{+} I^{-}\right)^{1 / 2} L\right]\left\langle\exp \left[i \phi(\mathbf{r})-i \phi\left(\mathbf{r}^{\prime}\right)\right]\right\rangle_{L} \\
& \times\left\langle\exp \left[i \Psi\left(\mathbf{r}^{\prime}\right)-i \Psi(\mathbf{r})\right]\right\rangle_{M} S^{*}(\mathbf{r}) S\left(\mathbf{r}^{\prime}\right),
\end{aligned}
$$

where $\langle\ldots\rangle_{L}$ and $\langle\ldots\rangle_{M}$ indicate the averaging operations over the fluctuations of the laser source and of the random medium, respectively, crossed by the signal before impinging upon the nonlinear medium in which phase conjugation takes place.

Equation (46) implies that $\Gamma\left(\mathbf{r}, \mathbf{r}^{\prime}\right)$ goes to zero whenever $\left|\mathbf{r}-\mathbf{r}^{\prime}\right|$ exceeds the smallest value between the transverse coherence length of the laser and the typical correlation scale of the random medium. This length sets the largest transverse dimension of the signal beam over which a goodfidelity phase conjugation can be achieved.

\section{APPENDIX A}

After multiplying both sides of Eqs. (6a) and (6b) by $\mathbf{E}(\xi, \sigma$; $\mathbf{r}) \exp \left[-i\left(\beta_{\xi}-k\right) z\right]$ and $\mathbf{E}(\xi, \sigma ; \mathbf{r}) \exp \left[i\left(\beta_{\xi}-k\right) z\right]$, respectively, and by integrating over the interval $\Delta_{s}$ we obtain, on the left-hand sides,

$$
\begin{aligned}
& \iint_{\Delta_{s}} \mathrm{~d} \xi \mathbf{E}(\xi, \sigma ; \mathbf{r}) \exp \left[\mp i\left(\beta_{\xi}-k\right) z\right] \mathrm{d} c^{ \pm}(\xi, \sigma ; z) / \mathrm{d} z \\
= & (\partial / \partial z) \iint_{\Delta_{s}} \mathrm{~d} \xi \mathbf{E}(\xi, \sigma ; \mathbf{r}) \exp \left[\mp i\left(\beta_{\xi}-k\right) z\right] c^{ \pm}(\xi, \sigma ; z) \\
\pm & i \iint_{\Delta_{s}} \mathrm{~d} \xi\left(\beta_{\xi}-k\right) \mathbf{E}(\xi, \sigma ; \mathbf{r}) \exp \left[\mp i\left(\beta_{\xi}-k\right) z\right] c^{ \pm}(\xi, \sigma ; z),
\end{aligned}
$$

which, taking advantage of the expansion

$$
\begin{aligned}
\beta_{\xi}-k=\left(k^{2}-\xi^{2}\right)^{1 / 2}-k & \cong k-\xi^{2} / 2 k+\ldots \\
& =-\xi^{2} / 2 k+\ldots,
\end{aligned}
$$

can be written in the form

$$
\begin{aligned}
& (\partial / \partial z) \epsilon_{s}^{\sigma, \pm} \mp(i / 2) \iint_{\Delta_{s}} \mathrm{~d} \xi\left(\xi^{2} / k\right) \mathbf{E}(\xi, \sigma ; \mathbf{r}) \\
& \quad \times \exp \left[\mp i\left(\beta_{\xi}-k\right) z\right] c^{ \pm}(\xi, \sigma ; z)+\ldots= \\
& \quad \times(\partial / \partial z) \epsilon_{s}{ }^{\sigma, \pm} \pm(i / 2 k) \nabla_{\mathbf{r}}{ }^{2} \epsilon_{s}{ }^{\sigma}+\ldots=L_{s}{ }^{ \pm} \epsilon_{s}{ }^{\sigma}
\end{aligned}
$$

\section{ACKNOWLEDGMENTS}

B. Crosignani gratefully acknowledges many helpful discussions with Paolo Di Porto. This research was supported by the National Science Foundation and by the U.S. Office of Naval Research.

* On leave from Fondazione Ugo Bordoni, Roma, Italy.

\section{REFERENCES}

1. See, for example, A. Yariv, "Phase-conjugate optics and real time holography," IEEE J. Quantum Electron. QE-14, 650-660 (1978).

2. A. Yariv, "On transmission and recovery of three-dimensional image information in optical waveguides," J. Opt. Soc. Am. 66, 301-306 (1976).

3. R. Hellwarth, "Theory of phase-conjugation by four-wave mixing in a waveguide," IEEE J. Quantum Electron. QE-14, 101-109 (1979).

4. B. Crosignani, "Self-induced nonlinear effects in optical fibers: a unified approach," in New Directions in Guided Waves and Coherent Optics, D. Ostrowsky and E. Spitz, eds. (Nijhoff, The Hague, 1984).

5. R. Trebino and A. E. Siegman, "Phase-conjugate reflection at arbitrary angles using $\mathrm{TEM}_{00}$ pump beams," Opt. Commun. 32, $1-4(1980)$.

6. E. Bochove, "Theory of phase conjugation by degenerate fourwave mixing using spatially varying pump beams," J. Opt. Soc. Am. 73, 1330-1342 (1983).

7. T. Wilson and L. Solymar, "Two-dimensional coupled differential equations for degenerate four-wave mixing," Appl. Phys. Lett. $38,669-670$ (1981). 
8. D. Marcuse, Theory of Dielectric Optical Waveguides (Academic, New York, 1978).

9. B. Crosignani and P. Di Porto, "Electromagnetic propagation in a turbulent medium: a new approach," J. Opt. Soc. Am. 73, 1581-1584 (1983).
10. A. Yariv, Quantum Electronics, 2nd ed. (Wiley, New York, 1975), Chap. 19.

11. D. M. Pepper and R. L. Abrams, "Narrow optical bandpass filter via nearly degenerate four-wave mixing," Opt. Lett. 3, 212-214 (1978). 